\title{
Time-resolved $x$-ray scattering studies on the BioCARS beam line at the APS: the nuts and bolts of pushing the limits achievable at a synchrotron facility P. Anfinrud ${ }^{1}$, F. Schotte ${ }^{2}$, H. S. Cho ${ }^{3}$, V. Stadnytskyi ${ }^{4}$ \\ ${ }^{1}$ National Institutes of Health ${ }^{2}$ National Institutes of Health, ${ }^{3}$ National Institutes of Health, ${ }^{4}$ National Institutes of Health anfinrud@nih.gov
}

Time-resolved x-ray measurements using the pump-probe technique afford time resolution down to the duration of the corresponding pump and probe pulses, and precision ultimately limited by the number of photons detected. Single $\mathrm{x}$-ray pulses, or short duration bursts of pulses arriving at a high repetition rate from a synchrotron facility can be isolated by a series of properly synchronized choppers and transmitted to the sample on demand by a fast millisecond shutter. When employing a synchronized short pulse laser as a pump, the time resolution achievable is limited only by the $\sim 100 \mathrm{ps}$ duration of the $\mathrm{x}$-ray pulse. To maximize flux, and therefore sensitivity, the $\mathrm{x}$-ray source is not passed through a monochromator, but instead its energy spectrum is kept suitably narrow for scattering studies by employing short period undulators. Though the average flux is significantly reduced by the need to gate the $\mathrm{x}$-ray transmission, the peak flux is quite high, and it has proven beneficial to rapidly translate the sample to a fresh location before the next pump-pulse pair arrives, which is readily accomplished with a home-built, high-speed xyz translation stage. A home-built field-programmable-gate-array (FPGA) was developed to properly synchronize the laser, $\mathrm{x}$-ray choppers and shutters, and sample position to allow rapid data collection of time-resolved, pump-probe $\mathrm{x}$-ray scattering images. The signal-to-noise ratio achieved in time-resolved studies following a temperature jump (T-jump) is limited by the magnitude of the T-jump, which depends on laser pulse energy and the volume to be heated. After acquiring a secondary K-B mirror pair to enable a tighter focus at the sample location, the volume of sample that needed to be heated decreased significantly, enabling $\sim 20^{\circ} \mathrm{C} \mathrm{T}$-jumps in under $10 \mathrm{~ns}$, and enabling time-resolved crystallography studies with significantly smaller crystals. The broad range of scattering angles and high flux available facilitate the acquisition of scattering images from crystallized biomolecules or biomolecules in solution at high spatial and time resolution. 